\section{Preference for dry sex, condom use and risk of STI among HIV-negative black women in the Western Cape province, South Africa}

\author{
Priscilla Reddy ${ }^{a}$, Dorina Saleh-Onoya ${ }^{a *}$, \\ Sibusiso Sifunda ${ }^{a}$, Delia Lang ${ }^{\mathrm{b}}$, Gina Wingood ${ }^{\mathrm{b}}$, \\ Bart van den Borne ${ }^{c}$ and Robert A.C. Ruiter ${ }^{d}$
}

\begin{abstract}
The practice of dry sex is reportedly common among young black women in South Africa. The aim of this study was to explore the relationship of women's preference for dry sex with condom use and the prevalence of Chlamydia trachomatis (CT), Neisseria gonorrhoea (NG) and Trichomonas vaginalis (TV) infections. Between January 2006 and December 2007, 446 women completed a behavioural survey in isiXhosa which assessed demographic information, sexual behaviours, condom use behaviour and other potential correlates. In total, 159 (36.72\%) women indicated preferring dry sex. A multivariate logistic regression model indicated that participants who preferred dry sex were more likely to report past STI episodes and to have a partner who also preferred dry sex. The findings indicate that dry sex behaviour was not directly associated with condom use and STI (CT, NG, and TV) prevalence but may have been associated with relationships in which sexual preferences of the male partner were dominant.
\end{abstract}

Key words: dry sex, gender role, sexual behaviour, condom use, sexually transmitted infections

The practice of wiping, douching or inserting substances into the vagina is reportedly common in sub-Saharan Africa. ${ }^{1-5}$ Intercourse following such vaginal treatment is referred to as 'dry sex $^{\prime} .{ }^{1,2}$ The most common reasons offered by women for these practices include the enhancement of sexual experience through sensations of vaginal dryness, tightness or warming; cleansing of the vagina before or after intercourse; treatment or prevention of STI; restoration and tightening of the vagina after delivery; or the satisfaction of a partner..$^{1-3}$ Although the evidence in the literature is inconclusive, ${ }^{1-4}$ it has been suggested that 'dry sex' may increase the risk of HIV infection among women through the sloughing of the vaginal wall during sex, or possible inflammation, ulceration or epithelial damage caused by the insertion of substances in the vagina.,

In a study conducted among Zimbabwean women, participants indicated that condoms frequently burst when used in conjunction with drying agents or 'because the vagina was so

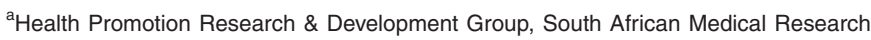
Council, P.O. Box 19070, Tygerberg, Cape Town 7505, South Africa.

${ }^{b}$ Department of Behavioral Science and Health Education, Rollins School of Public Health, Grace Crum Rollins Building, Emory University, 1518 Clifton Road, Atlanta, Georgia 30322, U.S.A.

'Department of Health Promotion and Health Education, Maastricht University, P.O. Box 616, 6200 MD, Maastricht, the Netherlands.

dDepartment of Work and Social Psychology, Maastricht University, P.O. Box 616, 6200 $\mathrm{MD}$, Maastricht, the Netherlands.

*Author for correspondence: E-mail:dorina.saleh@mrc.ac.za tight', and condoms may block the effect of some 'love agents' such as lovedrops (a commercial agent imported from South Africa) or mutundo wegudo (a traditional powder believed to have been made from baboon's urine, which when rubbed on incisions on the body is believed to magically cause a man to remain faithful to one woman). ${ }^{5}$ In South Africa, where the HIV prevalence among pregnant women attending public health services in 2006 was $29.1 \%$, ${ }^{6}$ there is increasing concern over the high prevalence of the practice of 'dry sex' ${ }^{1,7}$ It has been reported that $60 \%$ of men and $46 \%$ of interviewed women indicated preferring dry sex over lubricated sex. ${ }^{1}$ The most common materials used included tissues, towels or toilet paper; very few of the women reported using traditional herbs to dry the vagina. The specific materials or substances may vary widely across population groups. ${ }^{1,2}$

Many studies have focused on elucidating the mechanism through which substances inserted into the vagina, or dry sex practices, may directly increase the risk of HIV in women. ${ }^{1-5} \mathrm{~A}$ preference for the practice of dry sex among women could, however, reduce their desire to use condoms. Research into the implications of the preference for dry sex among women on their uptake of condoms use is therefore important. The objectives of this study were to describe dry sex behaviour among Xhosa women in South Africa, and to evaluate the association of the preference for dry sex with the rate of condom use and the prevalence of sexually transmitted infections (STIs) involving Chlamydia trachomatis (CT), Neisseria gonorrhoea (NG) and Trichomonas vaginalis (TV).

\section{Methods}

\section{Study setting and participants}

Data for this study were drawn from baseline measures of a randomised, controlled trial, which evaluated an HIV riskreduction intervention programme designed for isiXhosaspeaking women living in peri-urban areas of the Western Cape province, South Africa. This RCT is currently being completed. The Western Cape province is the second wealthiest province in South Africa. It is highly urbanised, with $87 \%$ of its population living in the Cape metropolis. Only $21 \%$ of the population is classified as black, $54 \%$ is 'coloured' and the rest is 'white'. ${ }^{8}$ There are, however, acute differences in health provision, health status and HIV prevalence that are closely related to racial, socioeconomic and type-of-locality differences. ${ }^{9}$ HIV prevalence is lowest among people living in wealthy formal urban settlements and highest in poor, informal urban settlements (referred to as townships), where at least $50 \%$ of adults are unemployed. ${ }^{9}$

Recruitment into the intervention study occurred between January 2006 and December 2007. Women were eligible to participate if they were black, isiXhosa-speaking women aged between 18 and 35, sought voluntary counselling and testing (VCT) services at any one of five peri-urban primary health care clinics, and had tested HIV-negative in the previous eight weeks. Participants were reassured that their identities would be treated as confidential.

\section{Data collection}

All 446 women gave written informed consent to complete an interviewer-administered behavioural questionnaire and provided one self-administered vaginal swab. Participants were guided to self-administer the vaginal swab in a sterile manner. ${ }^{10}$

The questionnaire was translated into isiXhosa by bilingual researchers and checked for linguistic appropriateness, comprehension and cultural relevance, and then translated back from 


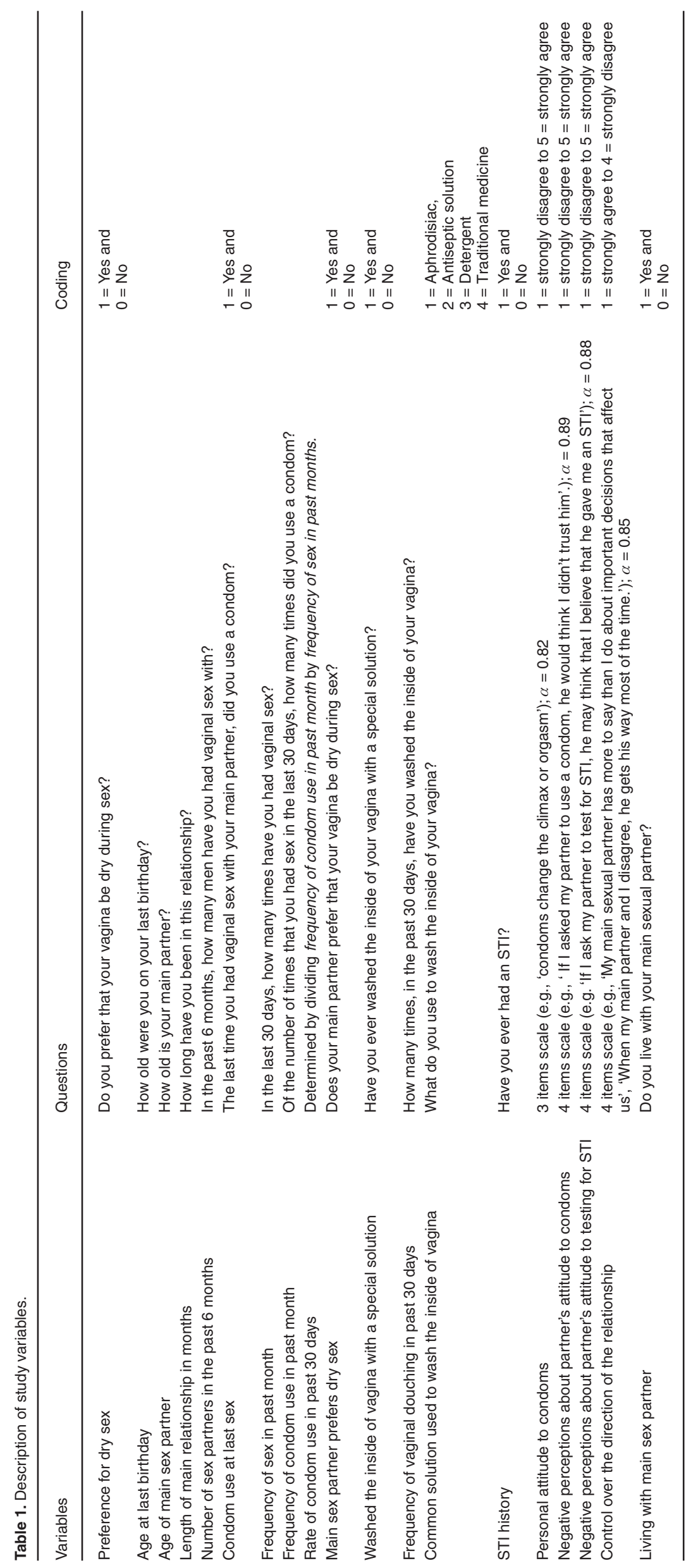

isiXhosa into English, to ensure accuracy and meaning of constructs. The questionnaires were computer-based (ACASI) and administered by trained, isiXhosa-speaking, black, female interviewers. This assessment procedure took about an hour to complete. Participants were given R20 (approx. \$2.74) for completing the assessment procedures.

All participants' vaginal swabs were tested for CT, NG, and TV. Tests for CT and NG were conducted using the COBAS Amplicor CT/NG PCR-based assay (Roche Molecular Diagnostics). ${ }^{11}$ The PCR test for TV was done using a PCR with the primers set TVK $3 /$ TVK $7 .{ }^{12}$ STI results were coded 0 for a negative result and 1 for a positive result. Those who tested positive for any one STI were referred to a contracted medical practitioner in their respective areas and were given single-dose prescriptions of azithromycin (1 g) for CT, ciprofloxacin (500 mg) for NG or metronidazole ( $2 \mathrm{~g}$ ) for TV infection..$^{13}$ The medical practitioner supervised the uptake of the treatment. The study protocol was approved by the Ethics Committee of the South African Medical Association (SAMA) and the Emory University Institutional Review Board (IRB) prior to implementation.

\section{Measures}

The variable 'Minimum of 1 STI' was created from the STI test results and denotes frequency of infection with at least one STI (coded Yes $=1$ and No = 0).

The questionnaire included measures of condom use at last sexual encounter, number of sex partners in the past six months, preference for dry sex, preference or otherwise of main partner for dry sex, frequency of sex in past month, frequency of condom use in the past month, rate of condom use, personal attitude towards condom use, perceived attitude of main partner towards condom use, perceived attitude of main partner towards STI testing, STI history, length of main relationship, whether living with main sex partner, use of vaginal douching and frequency thereof, solutions regularly used to wash the vagina, and control over the direction of the relationship. The measurement details of these variables are described in greater detail in Table 1.

\section{Data analysis}

Frequencies and percentages, or means and standard deviations (s.d.), were used to describe categorical and continuous variables, respectively. Logistic regression was used to evaluate univariate relationships with preference for dry sex. Variables with a significant univariate relationship with preference for dry sex were included in the multivariate regression model. The adjusted relationships of these measures with preference for dry sex were modelled in a multivariate logistic regression model. Significance level was set at the 5\% level for all analyses. All analyses were conducted in SPSS version 15 (SPSS Inc., Chicago, IL). 


\section{Results}

The demographic characteristics of the study sample are presented in Table 2 . The mean age of participants was 23 years. The majority $(88.42 \%)$ had reached secondary school, but only $25.84 \%$ had at least a Grade 12 level of education. Most women were unemployed $(75.28 \%)$ and financially dependent on their male partners $(30.11 \%)$, parents or grandparents $(26.97 \%)$, or other members of their families or friends $(12.36 \%)$.

\section{Univariate models}

In total, $159(36.72 \%)$ women indicated preferring dry sex. Of these, $135(84.91 \%)$ indicated that their partners also preferred dry sex. The results of the univariate test of independence between preference for dry sex and questionnaire variables and STI prevalence are presented in Table 3. Participants with a preference for dry sex were significantly older, more likely to have been living with their main male partner, more likely to indicate that their partner also preferred dry sex, and more likely to have had an STI in the past. Furthermore, participants who preferred dry sex had been in a relationship with their main partner for significantly longer periods, reported significantly lower control over the direction of their relationship, and had stronger negative perceptions about their partner's attitude towards condom use as well as testing for an STI. There was no univariate association between preference for dry sex and either the rate of condom use in past 30 days, condom use at last sex, or STI (CT, NG, and TV) prevalence.

\section{Multivariate model}

Variables that revealed significant univariate associations with preference for dry sex were further explored in a multivariate logistic model to determine whether these associations were unique and independent. The multivariate regression analysis modelled individual variables' associations with preference for dry sex while adjusting for the effect of interrelations between them. Among the variables that were entered in the multivariate
Table 2. Demographic profile of participants.

\begin{tabular}{lc} 
& $n(\%)$ \\
\hline Age, mean (s.d.) & $23.37(4.21)$ \\
Highest level of education & \\
Primary school & $51(11.56)$ \\
Secondary school & $276(62.59)$ \\
Matric & $102(23.13)$ \\
Tertiary & $12(2.72)$ \\
Employment status & \\
Employed & $96(21.92)$ \\
Unemployed & $335(76.48)$ \\
Self-employed & $7(1.60)$ \\
Main source of income & \\
Job & $77(17.30)$ \\
Child grant & $42(9.44)$ \\
Disability grant & $8(1.80)$ \\
Sexual partner & $120(26.97)$ \\
Parents or grandparents & $134(30.11)$ \\
Family members or friends & $55(12.36)$ \\
Other & $9(2.02)$ \\
\hline
\end{tabular}

model, only STI history, length of relationship and having a partner who preferred dry sex remained significantly associated with preference for dry sex. Women who preferred dry sex were more likely to indicate that their partner also preferred dry sex, more likely to have had a history of STI, and more likely to have been in the relationship for a longer period (see Table 3).

\section{Discussion}

The results of this study indicate that preference for dry sex is not directly related to either the rate of condom use in the past 30 days, condom use at last intercourse, or STI prevalence. The preference for dry sex may, however, affect condom use through partner-related variables that may have an impact on the adoption of condom use by women. In the univariate model, participants who preferred dry sex strongly indicated that their

Table 3. Description of study variables and results of univariate and multivariate logistic models with preference for dry sex as dependent variable.

\begin{tabular}{|c|c|c|c|c|c|c|c|c|}
\hline \multirow[b]{3}{*}{ Age at last birthday, mean (s.d.) } & \multicolumn{2}{|c|}{ Participant prefers dry sex } & \multicolumn{3}{|c|}{ Univariate model } & \multicolumn{3}{|c|}{ Multivariate model } \\
\hline & \multirow{2}{*}{$\frac{\text { No }}{22.99(3.79)}$} & \multirow{2}{*}{$\frac{\text { Yes }}{24.13(4.77)}$} & \multirow{2}{*}{$\frac{\text { OR }}{1.07}$} & \multicolumn{2}{|c|}{$95 \% \mathrm{Cl}$} & \multirow{2}{*}{$\frac{\mathrm{OR}}{1.00}$} & \multicolumn{2}{|c|}{$95 \% \mathrm{Cl}$} \\
\hline & & & & 1.02 & 1.12 & & 0.93 & 1.08 \\
\hline Age of main sex partner, mean (s.d.) & $27.27(5.63)$ & $28.27(7.66)$ & 1.02 & 0.99 & 1.06 & & & \\
\hline Length of main relationship in months, mean (s.d.) & $44.98(34.55)$ & $56.98(52.50)$ & 1.01 & 1.00 & 1.01 & 1.01 & 1.00 & 1.01 \\
\hline Number of sex partners in the past 6 months, mean (s.d.) & $1.08(0.33)$ & $1.06(0.30)$ & 0.78 & 0.41 & 1.51 & & & \\
\hline Condom use at last sex $(n, \%)$ & $63(23.00)$ & $30(18.90)$ & 0.78 & 0.48 & 1.27 & & & \\
\hline Main sex partner prefers dry sex, $n(\%)$ & $40(14.9)$ & $135(86.00)$ & 34.97 & 19.94 & 61.36 & 36.81 & 20.07 & 67.52 \\
\hline Washed the inside of vagina with a special solution, $n(\%)$ & $40(14.6)$ & $30(18.9)$ & 1.36 & 0.89 & 2.29 & & & \\
\hline Frequency of vaginal douching in past 30 days, mean (s.d.) & $6.83(10.07)$ & $6.88(8.43)$ & 1.00 & 0.95 & 1.05 & & & \\
\hline \multicolumn{9}{|l|}{ Common solution used to wash the inside of vagina } \\
\hline Aphrodisiac, $n(\%)$ & $1(0.40)$ & 0 & NA & & & & & \\
\hline Antiseptic solution, $n(\%)$ & $35(12.80)$ & $26(16.40)$ & 1.00 & 0.21 & 4.85 & & & \\
\hline Detergent, $n(\%)$ & 0 & $1(0.60)$ & NA & & & & & \\
\hline CT positive, $n(\%)$ & $73(27.97)$ & $48(30.77)$ & 1.15 & 0.74 & 1.77 & & & \\
\hline NG positive, $n(\%)$ & $32(12.26)$ & $21(13.46)$ & 1.11 & 0.62 & 2.01 & & & \\
\hline TV positive, $n(\%)$ & $89(34.10)$ & $57(36.54)$ & 1.11 & 0.74 & 1.68 & & & \\
\hline Positive for at least one STI, $n(\%)$ & $140(53.6)$ & $89(57.1)$ & 1.15 & 0.77 & 1.71 & & & \\
\hline STI history, $n(\%)$ & $183(66.8)$ & $123(77.4)$ & 1.70 & 1.09 & 2.66 & 2.01 & 1.04 & 3.90 \\
\hline Personal attitude to condoms, mean (s.d.) & $2.41(1.10)$ & $2.47(1.12)$ & 1.02 & 0.96 & 1.08 & & & \\
\hline Negative perceptions about partner's attitude to condoms, mean (s.d.) & $2.29(1,16)$ & $2.58(1.18)$ & 1.05 & 1.01 & 1.90 & 1.00 & 0.93 & 1.07 \\
\hline Negative perceptions about partner's attitude to testing for STI, mean (s.d.) & $2.51(1.02)$ & $2.78(1.11)$ & 1.05 & 1.01 & 1.10 & 1.03 & 0.96 & 1.10 \\
\hline Control over the direction of the relationship, mean (s.d.) & $2.70(0.48)$ & $2.52(0.57)$ & 0.85 & 0.77 & 0.94 & 1.00 & 0.87 & 1.17 \\
\hline Living with main sex partner, $n(\%)$ & $48(17.5)$ & $45(28.3)$ & 1.86 & 1.17 & 2.96 & 1.10 & 0.54 & 2.25 \\
\hline
\end{tabular}


partner also preferred dry sex, perceived highly negative partner attitudes towards both condom use and STI tests, indicated having little control over the direction of their relationship, and were more likely to have a history of STI. When these associations were tested in a multivariate logistic model, only three variables remained significant: women who preferred dry sex were more likely to have had a partner who preferred dry sex, more likely to have had a history of STI, and more likely to have been in the relationship for a long period.

A review of the literature shows that the practice of dry sex has been previously associated with an STI history among men, who were likely to indicate as reasons for practicing dry sex, enjoyment, 'feeling well', and pleasure. Women, on the other hand, have reported participating in dry sex mainly to satisfy their partners. $^{1-3}$

We postulate that the risk of STI associated with dry sex may be related to the nature of the relationship in which it is practised, and as such may be an indicator for a relationship in which the male partner's sexual preferences are dominant. Since no data on the subject of dry sex were collected among men in this study, it is difficult to determine the position of the (main) male partner on dry sex. Furthermore, men's preference for dry sex and their attitude towards STI testing and condom use were measured with single-item variables that only expressed the perceptions of the women on these issues, and should be interpreted cautiously. Since the measure of STI history was obtained through self-reports of past STI episodes, this is not a strong enough variable to test the association between preference for dry sex and STI risk.

The interpretation of the results is limited to the population in which the research was done, and further research should be extended to men and women of different populations. The results provide no conclusive evidence for an association between women's preference for dry sex and condom use, and highlight the need for further research in the characteristics of relationships within which dry sex occurs, men's position on the practice of dry sex, and the implications for condom use, preferably using a longitudinal design to make causal inferences possible.

This study was supported with NIH funding (grant \#3P30 AI050409-06SI).

Received 17 November 2008. Accepted 25 February 2009.

1. Beksinska M.E., Rees H.V., Kleinschmidt I. and McIntyre J. (1999). The practice and prevalence of dry sex among men and women in South Africa: a risk factor for sexually transmitted infections? Sex. Transm. Infect. 75, 178-180.

2. Myer L., Kuhn L., Stein Z.A., Wright T.C. Jr. and Denny L. (2005). Intravaginal practices, bacterial vaginosis, and women's susceptibility to HIV infection: epidemiological evidence and biological mechanisms. Lancet Infect. Dis. 5, 786794

3. Brown J.E. and Brown R.C. (2000). Traditional intra-vaginal practices and the heterosexual transmission of disease: a review. Sex. Transm. Dis. 27, 183-187.

4. Brown R.C., Brown J.E. and Ayowa O.B. (1993). The use and physical effects of intravaginal substances in Zairian women. Sex. Transm. Dis. 20, 96-99.

5. Civic D. and Wilson D. (1996). Dry sex in Zimbabwe and implications for condom use. Soc. Sci. Med. 42, 91-98.

6. Department of Health of South Africa (2007). National HIV and syphilis antenatal sero-prevalence survey in South Africa 2007. Department of Health, Pretoria.

7. Baleta A. (1998). Concern voiced over 'dry sex' practices in South Africa. Lancet $352,1292$.

8. Shaikh N. and Adendorff T. (2000). Health Status Report. Health Status and Health Service Evaluation Report 1999-2000. Western Cape Department of Health, Cape Town.

9. Shaikh N., Abdullah F., Lombard C.J., Smith L., Bradshaw D. and Makubalo L. (2006). Masking through averages - intraprovincial heterogeneity in HIV prevalence within the Western Cape. S. Afr. Med. J. 96, 539-543.

10. Van de Wijgert J., Altini L., Jones H., de Kock A., Young T., Williamson A-L., Hoosen A. and Coetzee N. (2006). Two methods of self sampling compared to clinician sampling to detect reproductive tract infections in Gugulethu, South Africa. Sex. Transm. Infect. 33, 516-523.

11. Crotchfelt K.A., Welsh L.E., Debonville D., Rosenstraus M. and Quinn T.C. (1997). Detection of Neisseria gonorrhoeae and Chlamydia trachomatis in genitourinary specimens from men and women by a co-amplification PCR assay. J. Clin. Microbiol. 35, 1536-1540.

12. Crucitti T., Van Dyck E., Tehe A., Abdellati S., Vuylsteke B., Buve A. and Laga M. (2003). Comparison of culture and different PCR assays for detection of Trichomonas vaginalis in self collected vaginal swabs specimen. Sex. Transm. Infect. 79, 393-398.

13. Pepin J. and Mabey D. (2003). Sexually transmitted infections in Africa: single dose treatment is now affordable. Sex. Transm. Infect. 79, 432-434. 